\title{
Research of antenna for microwave energy transmission system for IOT
}

\author{
Liu NanPing ${ }^{1, a}$, Li Na ${ }^{2, b^{*}}$, Liu Song ${ }^{3, c}$ and Liu ChunQia ${ }^{4, d}$ \\ ${ }^{1}$ Tianjin Electronic Information College, No.4, Ya Shen road, Jin Nan District, Tianjin, China \\ 2 Tianjin Electronic Information College, No.4, Ya Shen road, Jin Nan District, Tianjin, China \\ ${ }^{3}$ Tianjin Electronic Information College, No.4, Ya Shen road, Jin Nan District, Tianjin, China \\ ${ }^{4}$ Tianjin Electronic Information College, No.4, Ya Shen road, Jin Nan District, Tianjin, China \\ aliunanping05@126.com, ${ }^{\mathrm{b}} 15302008723 @ 163 . c o m,{ }^{\mathrm{C}} T$ jliusong@126.com, \\ dliuchunqia2000@163.com
}

Keywords: IOT, antenna array, microstrip slot antenna, wireless sensor network.

Abstract. Wireless energy transmission technology is an important research field in recent years, and has a good research value and application prospect. Wireless energy transmission technology: short electromagnetic inductive coupling transmission technology, medium range of electromagnetic coupling resonance transmission technology, remote microwave power transmission technology. These three types of wireless energy transmission technology work in different application fields.

Through this article, we are to study the wireless energy transmission system, which is based on the non licensed $2.45 / 5.8 \mathrm{GHz}$ microwave, as the energy carrier, which can improve the transmission efficiency and realize the variable transmission of energy. The dual band $(2.45 / 5.8 \mathrm{GHz})$ segment is achieved by loading microstrip branch and slot, and the narrow band gap of microstrip slot antenna is solved.

The frequency of energy transmission is the same as that of the wireless sensor network. It can realize the reuse of the energy receiving system and the antenna. It can reduce the volume of the product and reduce the complexity of the system.

The research can be extended to other areas: for all kinds of small electronic products in the "free space", such as a variety of small aircraft, such as a variety of small aircraft, etc., for the "No power facility" family, office, etc..

\section{Preface}

IOT is a kind of Internet between things and things, which is the third change of the world information industry after the computer and the Internet. It refers to the various kinds of information sensor (equipment), real-time acquisition of information that require monitoring, connection, interactive objects or processes, etc., then become a huge network works with Internet. Its purpose is to realize the connection between the object and the person, the object and the object, all the goods and the network, and then to facilitate the identification, control and management ${ }^{[1]}$.

Therefore, the development and the application of the IOT will bring huge change to the daily life, industrial and agricultural production, and military. The perception of the IOT "organ" - wireless sensor is an essential component of the $\mathrm{IOT}^{[2-3]}$. How to provide energy for the number of sensors, a wide variety of functions and different functions, will be a challenge for the designing and planning of the IOT.

First of all, the "wireless" characteristics of the wireless sensor make it impossible to supply the power through the traditional wire.

Secondly, if the battery is applied to power a wireless sensor, to ensure the sensor's normal work, frequent examination and change would be happened on the sensors distributed in different network nodes. Moreover, there are many sensors that may be distributed in the areas that are difficult to maintain, such as narrow space, airtight place, water, fine pipe etc ${ }^{[4-5]}$.

So, if the energy is provided by the wireless energy transmission, and with the large capacity storage device and battery detection device, it can solve the above problems, and realize the automatic 
charging and maintenance of the sensor, thus greatly reducing the workload of the Internet of things and improving the reliability of the network ${ }^{[6-7]}$.

In addition, compared with the traditional wired energy transmission, wireless energy transmission technology has the following advantages:

(1) In the flammable, explosive, high temperature and other harsh environment, wireless energy transmission can avoid the electric spark, line aging and other security risks

(2)In the long distance energy transmission, it can save the transmission line and reduce the cost.

(3)Wireless energy transmission can avoid complicated wiring work, save the space required for wiring, simplify the installation of the system and reduce the volume of the system.

The research of wireless power transmission is very important for the development of the IOT technology and the practical application. In particular, the microwave energy transmission of antenna technology is becoming a hot spot and focus of research in academia and industry.

\section{The System Model of Microwave wireless energy transmission based on 2.45/5.8GHz.}

On one hand, depend on the hard efforts in academia and industry recent years, (such as NOKIA, LG, etc. they introduced the wireless charging mobile phone based on the principle of electromagnetic induction), the public's view had been successfully attracted to wireless energy transmission technology. However, no matter the electromagnetic resonance technology or the inductive coupling technology, they all applied in short-distance, and high power field. It still has the gap from the real "wireless charging". So, these techniques can't provide the function application of delivering energy to a large, widely distributed and small size wireless sensor.

On the other hand, the technical solution that use electromagnetic wave as the carrier of wireless energy transmission technology had been attract attention from researcher because of its long transmission distance, and a considerable number of research achievement have been obtained. To use electromagnetic wave to transmit energy, it must first solve the problem of the direction spread of the energy. Most of the research in China or abroad, the researchers use horn antenna instead of omnidirectional antenna to ensure the emitted energy concentrated in one direction, decrease the energy in the other direction loss ${ }^{[8]}$. But it needs for manual intervention or mechanical adjustment when emits to other direction.

The array antenna is the combination of more than two antenna elements, and the signal of the array antenna is also the synthesis of the transmitted signal of all antenna elements. The intensity and direction of the antenna can be adjusted conveniently by adjusting the composition of the array antenna. The performance of array antennas is needed for flexible wireless energy transmission systems.

At present, the majority of the array antenna used in wireless energy transmission is focused on the energy transmission between the solar satellite and the ground ${ }^{[9]}$. And its application in near distance (relative to the satellite and the ground of the distance in the case) developed due to the rapid developed of wireless sensor network technology (the important part of the technology of the Internet of things) and consumer electronics products market.

In this article, it proposes a wireless energy transmission system based on $2.45 / 5.8 \mathrm{GHz}$ microwave, which brings new features.

(1) the array antenna is used as an energy transmitting device to improve the transmission efficiency and realize the variable transmission;

(2)It's the first time to use the non licensed $2.45 / 5.8 \mathrm{GHz}$ microwave as an energy transfer vector, it lays a good foundation to the industrialization.

(3) $2.45 / 5.8 \mathrm{GHz}$ microwave is chosen as the energy carrier, and the same communication frequency with the wireless sensor network can be used to realize the reuse of energy receiving system and communication system, which can reduce the volume of the product and reduce the complexity of the system. This is not available in the microwave wireless power transmission system, which works in other frequency bands. 
(4) A dual band $(2.45 / 5.8 \mathrm{GHz})$ microstrip fed slot receiving antenna for wireless energy harvesting is proposed. The dual band $(2.45 / 5.8 \mathrm{GHz})$ segment is achieved by loading microstrip branch and slot, and the narrow band gap of microstrip slot antenna is overcome.

$2.45 / 5.8 \mathrm{GHz}$ microwave energy transmission system is shown in figure 1 .

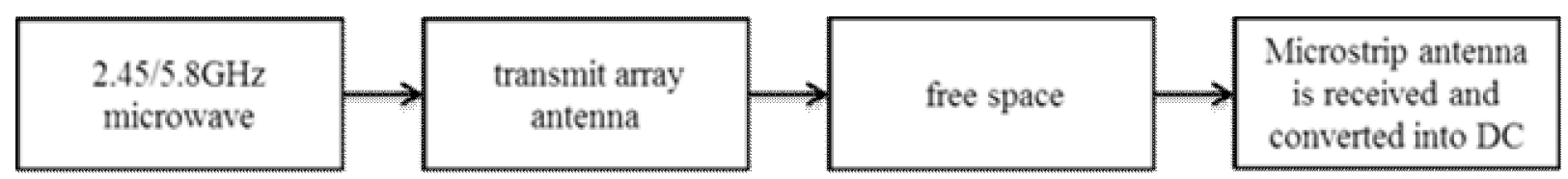

Fig 1. 2.45/5.8GHz microwave energy transmission system

\section{Design of the transmitting and receiving antennas based on $2.45 / 5.8 G H z$}

The most critical part in wireless energy transmission is the analysis and design of the transmitting and receiving antennas, especially receiving antennas more important.

On the one hand, the selection of antenna elements (array elements) in the antenna array, the design of the antenna element and array layout are studied. A total area of the antenna array and its beam synthesis method are designed and fabricated, which enable it to project electromagnetic wave (energy) in different directions ${ }^{[10]}$.

On the other hand, research and select the appropriate receiving antenna type, then design, manufacture receiving antenna, so that the receiving efficiency of the electromagnetic wave is as high as possible.

After the selection of transmiting and receiving antenna type, the ADS simulation software is used to simulate the electromagnetic field, to make the actual antenna system and measure the parameters.

\section{Design of transmit array antenna}

The direction of a single antenna is limited, for more suitable energy transmission, it will work at the same frequency of two or more single antenna, according to a certain requirement for feed and space array to make up the antenna array (it also called antenna array, antenna array antenna radiation unit called array element), the transmitter signal is also the synthesis of antenna unit.

The working principle of the transmit antenna array is: when the electromagnetic wave is transmitted to the same area of two or two columns, the electromagnetic wave will produce vector superposition, which is related to the amplitude and phase difference of each column.

The phase of the electromagnetic wave includes: the first phase, the time phase, the space phase. If the transmit antenna and its working frequency are determined, then the initial phase is determined, and the time phase is also determined by the time of the encounter of the electromagnetic wave. Because of the different location of the antenna array, the electromagnetic wave transmitted by the electromagnetic wave to the same receiving area is different, and the space phase value is different. In this way, several columns of electromagnetic wave in the meeting area with phase superposition, total field intensity increases, anti phase superposition, the total field will weaken. If the enhanced and weakened regions of the total electric field are relatively fixed in space, the radiation field structure of a single antenna is equivalent to that of an antenna array. Obviously, the intensity and direction of the antenna can be adjusted conveniently by adjusting the power of the array antenna. The performance of array antennas is needed for flexible wireless energy transmission systems.

\section{Design of receiving antenna}


Microstrip antenna is low cost, light weight, and has many other advantages, but the narrow band limits its practical application, by increasing parasitic elements or with different shape slot rectangular patch element can overcome the defects which microstrip antenna with narrow band characteristics, only single frequency operation and others.

This paper presents a dual band $(2.45 / 5.8 \mathrm{GHz})$ microstrip fed slot receiving antenna for wireless energy harvesting. The band $(2.45 / 5.8 \mathrm{GHz})$ segment is achieved by loading microstrip branch and dual slot, and the narrow band gap of microstrip slot antenna is overcomed. Through the ADS simulation, the variation of the working frequency of the slot antenna with the slot size parameters is obtained ${ }^{[11]}$.

\section{Design of slot antenna}

The transmission mode approximation method is used to design of microstrip antenna in the engineering, the input impedance $Z_{\text {in }}$ of the microstrip antenna at the resonant frequency, according to the theory of cavity model:

$$
Z_{\text {in }}=R+j X_{r}+j X_{f}
$$

In the formula (1), $X_{r}$ is the "resonance" reactance of the equivalent circuit for the parallel resonant circuit of the mode, ${ }^{X_{f}}$ is Synthesis (reactive) effect for other modes. The characteristic equation of the resonance frequency is

$$
X_{r}+X_{f}=0
$$

If the microstrip antenna is loaded with a reactance $X_{L}$, The characteristic equation of the formula (2) is changed to

$$
X_{r}+X_{f}+X_{L}=0
$$

By adjusting the value of $X_{L}$, two zero point can be obtained to achieve dual frequency (such as 2.45/5.8GHz) segment work.

In this paper, a dual band $(2.45 / 5.8 \mathrm{GHz})$ microstrip fed slot antenna structure is proposed, which is shown in Figure 2. The microstrip antenna is used in the Top layer, which can not only obtain a wide bandwidth, but also have good impedance matching performance over a wide frequency range.

In addition, if the Bottom layer (layer) of the antenna is etched into two slots (rectangular, as shown in Figure 3), which can be achieved by adjusting the relative position of the gap and the microstrip line and the size of the gap to achieve the best matching. The Bottom layer is etched into the two slot, which is equivalent to the introduction of two reactance, so that the microstrip antenna has two resonance points ${ }^{[12]}$.

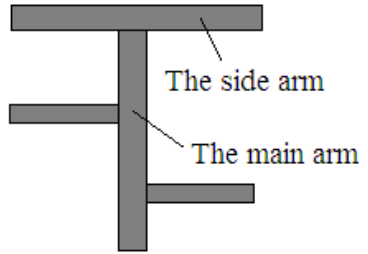

Fig2. Antenna (Top layer) structure model

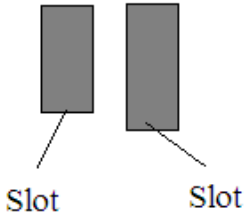

Fig.3 Antenna (Bottom layer) etching two slots

Considering the impedance matching of the interface, the width of the microstrip line can be calculated according to the empirical formula (4).

$$
Z_{\text {om }}=\frac{60}{\sqrt{\varepsilon_{f}}} \ln \left(\frac{8 h}{w}+\frac{w}{4 h}\right)
$$

In the formula (4), ${ }^{\varepsilon_{f}}$ is the equivalent permittivity (as known). 
According to the formula (4), if the characteristic impedance of the main arm of the branching type microstrip line is $50 \Omega$, its corresponding width was calculated to be $3 \mathrm{~mm}$; and the characteristic impedance of the side arm is $100 \Omega$, and the corresponding width was calculated to be $1.4 \mathrm{~mm}$.

\section{Parameters analysis and optimization of microstrip receiving antenna}

The physical dimension parameters of the antenna (Top) structure model in figure 2 are defined as shown in figure 4 . The parameters of the microstrip antenna are analyzed and optimized using ADS to obtain the various geometric parameters of the dual frequency $(2.45 / 5.8 \mathrm{GHz})$ for the wireless energy collection.

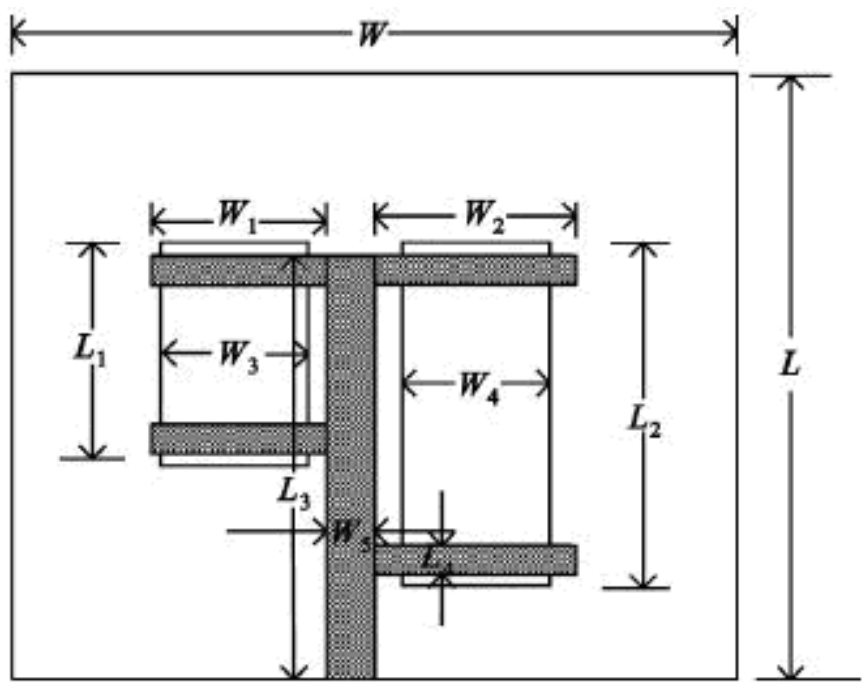

Fig4. Definition of physical dimension parameters

The simulation results show that the variation of the parameters L1, L2, W3 and W4 in Figure 4 has great influence on the return loss of the antenna ${ }^{[12]}$. So the parameters of L1, L2, W3 and W4 are selected to analyze the parameters of them.(In the analysis, each parameter selects an initial value, and when a parameter changes, the other parameters remain unchanged).

The initial parameters of the microstrip antenna are shown in Table 1.

Table1. The initial parameters of the microstrip antenna

\begin{tabular}{|c|l|l|l|l|l|l|l|l|l|l|l|}
\hline parameters & W & L & W1 & L1 & W2 & L2 & W3 & L3 & W4 & L4 & W5 \\
\hline $\begin{array}{c}\text { Size } \\
(\mathrm{mm})\end{array}$ & 55 & 55 & 18.2 & 20.2 & 17.9 & 40.3 & 9.2 & 42.5 & 12.1 & 3.2 & 4.1 \\
\hline
\end{tabular}

The effect of L1 on the return loss of the antenna is shown in Figure 5. Other parameters remain unchanged, the L1 size selected from $20.2 \mathrm{~mm}$ by $1 \mathrm{~mm}$ increased, the simulation results in Figure 5show that: in the low frequency range, with the increase of L1, resonant point shifted to the right; L1 is $21.2 \mathrm{~mm}$, return loss minimum; in the high frequency range, with the increase of L1, resonant point shifts to the left, return loss is reduced, bandwidth with reduced. 


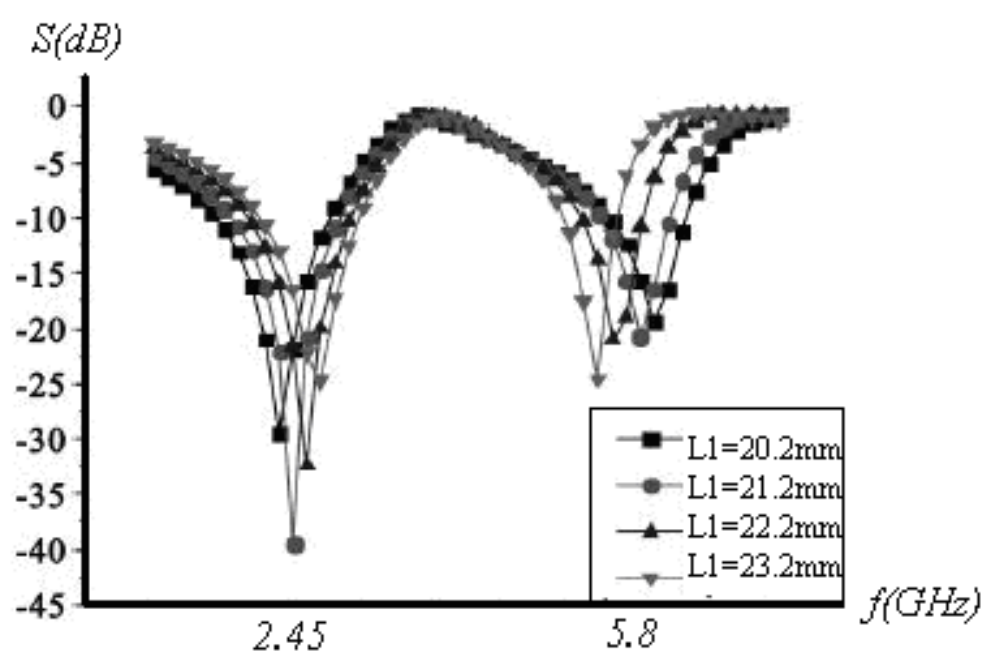

Fig5. The effect of $\mathrm{L} 1$ on the return loss of antenna

The effect of L2 on the return loss of the antenna is shown in Figure 6. Other parameters remain unchanged, the L2 size selected from $40.3 \mathrm{~mm}$ by $1 \mathrm{~mm}$ increased, the simulation results in Figure 6 show that: In the low frequency range, the return loss and the bandwidth increases with the decrease of L2, the resonant point remain unchanged; In the high frequency range, with the increase of L2, the frequency of the resonant frequency shift to left, the return loss is small, and the antenna impedance matching is worse.

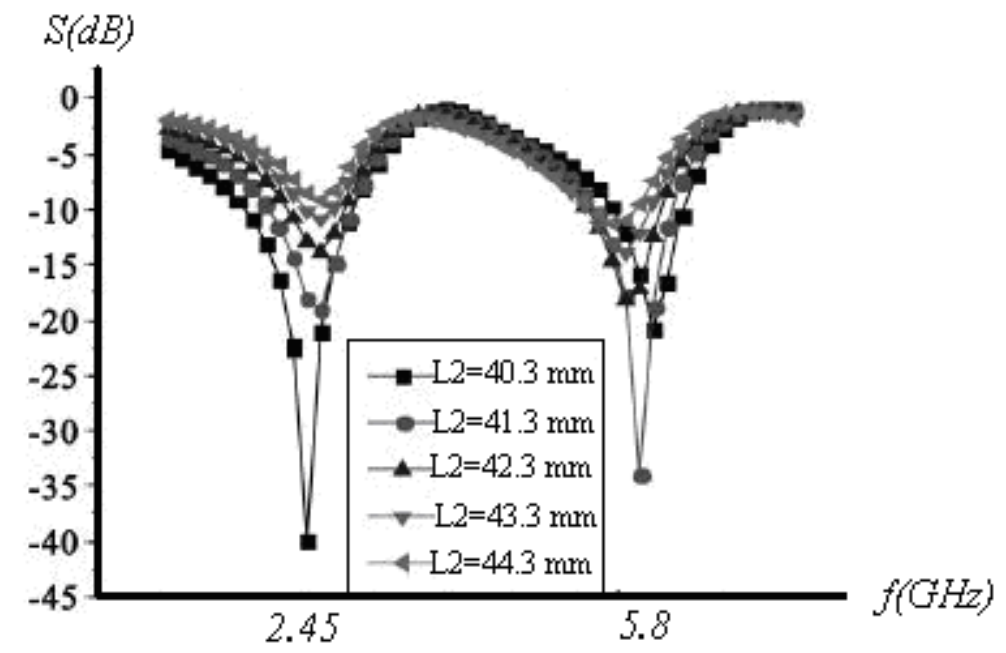

Fig6. The effect of L2 on the return loss of antenna

The effect of $\mathrm{W} 3$ on the return loss of the antenna is shown in Figure 7. Other parameters remain unchanged, the W3 size selected from $9.2 \mathrm{~mm}$ by $1 \mathrm{~mm}$ increased, the simulation results in Figure 7 show that: In the low frequency range, the W3 is almost no effect. In the high frequency range, the resonant point shift to left, the bandwidth and the return loss are almost unchanged with the increase of W3. 


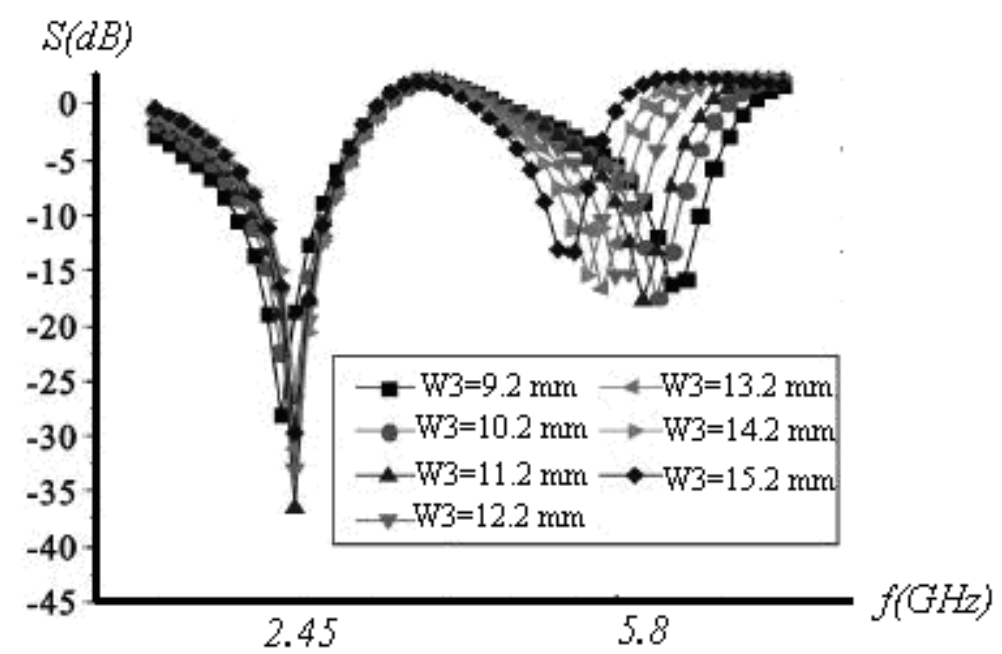

Fig7. The effect of $\mathrm{W} 3$ on the return loss of antenna

The effect of W4 on the return loss of the antenna is shown in Figure 8. Other parameters remain unchanged, the W4 size selected from $12.1 \mathrm{~mm}$ by $1 \mathrm{~mm}$ increased, the simulation results in Figure 8 show that: In the low frequencies and high frequencies, with the increase of $\mathrm{W} 4$, the resonant point shift to right, the greater the return loss, and the bandwidth is increased, the better matching performance is ${ }^{[13]}$.

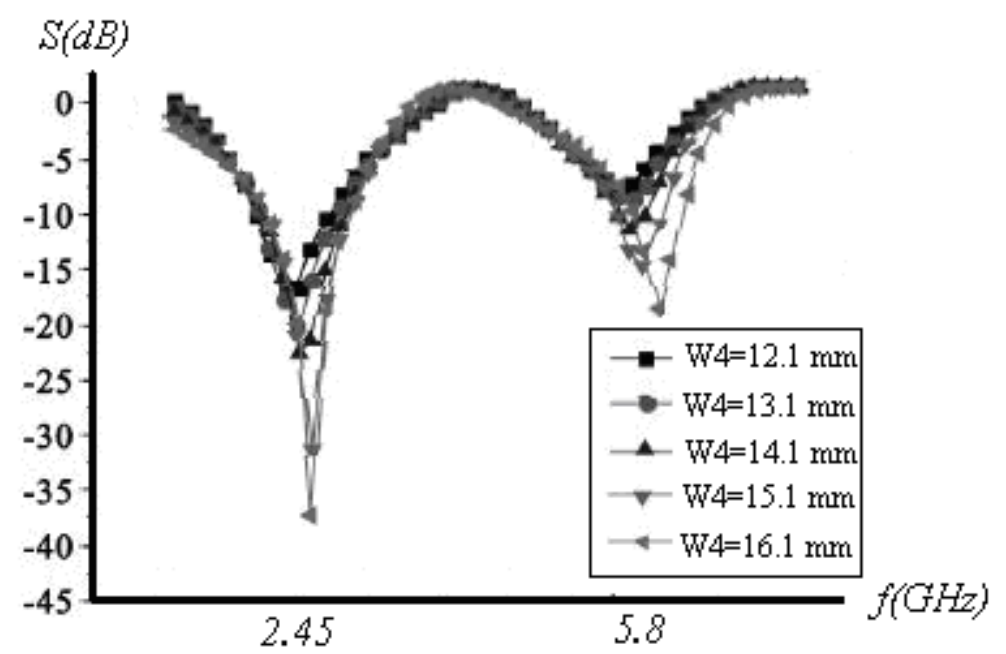

Fig8. The effect of W4 on the return loss of antenna

The simulation results show that: Adjust the slot size can change the distance between the two resonant frequency. The optimized parameters of the microstrip antenna are shown in Table 2.

Table2. The optimized parameters of the microstrip antenna

\begin{tabular}{|c|c|c|c|c|c|c|c|c|c|c|c|}
\hline Parameters & W & L & W1 & L1 & W2 & L2 & W3 & L3 & W4 & L4 & W5 \\
\hline $\begin{array}{c}\text { Size } \\
(\mathrm{mm})\end{array}$ & 55 & 55 & 18.2 & 23.2 & 17.9 & 40.3 & 11.2 & 42.5 & 16.1 & 3.2 & 4.1 \\
\hline
\end{tabular}

When the resonant frequency is $2.45 \mathrm{GHz}$ and $5.8 \mathrm{GHz}$, the gain direction is shown in Figure 9 (a) and (b). As can be seen from Figure 9, the antenna has a certain direction, which can be used to receive microwave energy ${ }^{[14-15]}$. 


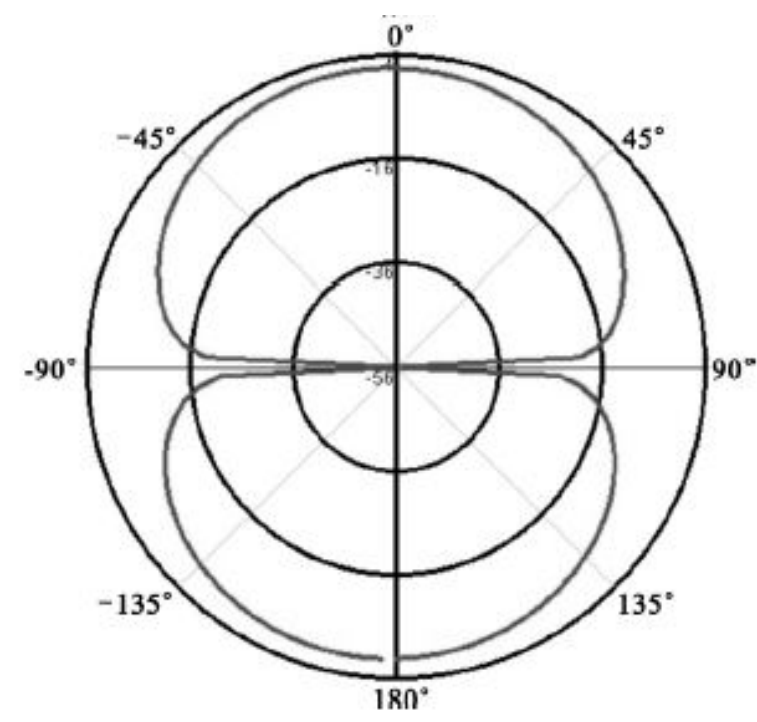

(a) The gain of the antenna when the resonant frequency is $2.45 \mathrm{GHz}$

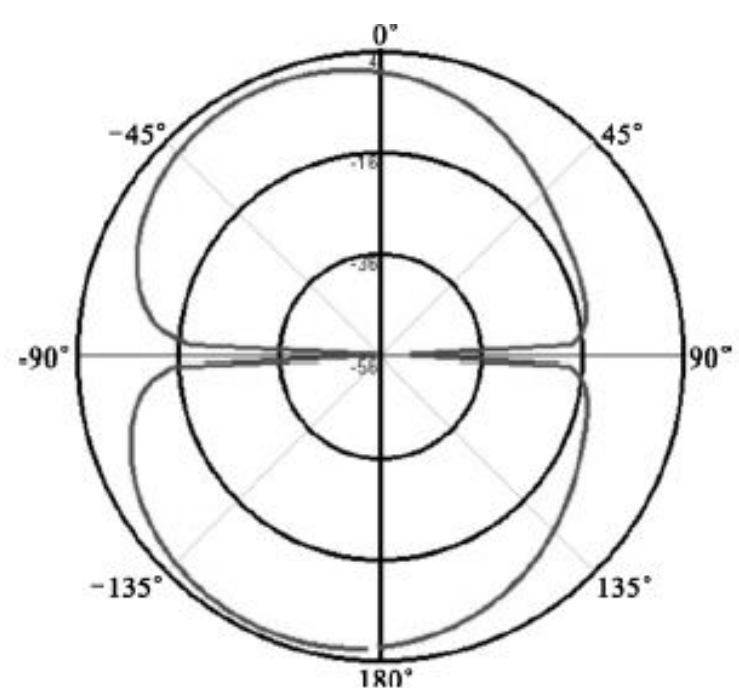

(b) The gain of the antenna when the resonant frequency is $5.8 \mathrm{GHz}$

Fig9. The gain direction map when the resonant frequency is $2.45 \mathrm{GHz}$ and $5.8 \mathrm{GHz}$

\section{Summary}

The reuse of the energy receiving system and the communication system is helpful to the miniaturization of the products and the complexity of the system.

Using array antenna as the energy transmitting device, it can improve the energy transfer efficiency and the variable transmission of energy. After the completion of the design and production of the transmit antenna array, the transmission efficiency of the transmitting antenna is measured, and the transmission efficiency is improved by $9 \%$.

Slot microstrip antenna is suitable for the environment of wireless dual band $(2.45 / 5.8 \mathrm{GHz})$ energy receiving, small size, low cost, more practical. A standard gain horn antenna is designed and fabricated, which is used in the microwave power test to test the receiving efficiency of the receiving antenna, and the receiving efficiency is improved by $11 \%$.

The research results of this paper will be of great significance to the practical application and industrialization of the Internet of things technology.

\section{References}

[1] Shen Bin. The concept model and architecture of the IOT[J]. Journal of Nanjing University of Posts and Telecommunications(Natural Science). 2014,05:8-9.

[2] Huang Xiao Li. Wireless energy transmission technology[J]. Wireless technology. Wireless Internet technology.

[3] Zhang Hua, Chen Hong. Design of wireless energy transfer model and experimental device[J]. Journal of Wuhan University of Technology Information \& Management Engineering. 2014,05:33-68

[4]Su Cheng. The Framework of IOT[J]. ZTE TECHNOLOGY JOURNAL. 2011(01)

[5] Huang Ling. $5.8 \mathrm{GHz}$ microwave energy transmission system for micro robot[J].Robot. 2011,04:509-543.

[6] Li Tao. Research status and application prospect of wireless transmission technology[J]. Science \& Technology Information. 2014(9) 
[7]Liu Ling, Guo Qing Gong. The 2.45/5.8GHz microstrip patch antenna for wireless transmission. Information and electronic engineering. 2009,03:101-125.

[8]Zhao Cheng. Research on wireless energy projection technology[D]. (Shenyang Ligong University. 2011.

[9] Shinohara, N. Development of high efficient phased array for microwave power transmission of Space Solar Power Satellite/Station. Antennas and Propagation Society International Symposium (APSURSI), 2010 IEEE

[10] Chun-Chih Lo ; Yu-Lin Yang ; Chi-Lin Tsai ; Chieh-Sen Lee ; Chin-Lung Yang. Novel wireless impulsive power transmission to enhance the conversion efficiency for low input power. Microwave Workshop Series on Innovative Wireless Power Transmission: Technologies, Systems, and Applications (IMWS), 2011 IEEE MTT-S International

[11] Kuo, F.-M. ; Shi, J.-W. ; Shao-Ning Wang ; Nan-Wei Chen ; Po-Tsung Shih ; Chun-Ting Lin ; Wen-Jr Jiang ; Er-Zih Wong ; Chen, J. ; Sien Chi. W-Band Wireless Data Transmission by the Integration of a Near-Ballistic Unitraveling-Carrier Photodiode With a Horn Antenna Fed by a Quasi-Yagi Radiator. Electron Device Letters, IEEE Volume: 30 , Issue: 10

[12] Massa, A. ; Oliveri, G. ; Viani, F. ; Rocca, P. Array Designs for Long-Distance Wireless Power Transmission: State-of-the-Art and Innovative Solutions. Proceedings of the IEEE Volume: 101, Issue: 6

[13] Y. Li, and V. Jandhyala, "Design of retrodirective antenna arrays for short-range wireless power transmission," IEEE Trans. Antennas Propag ., vol. 60, no. 1, pp. 103-266, Jan. 2012

[14] Inoue, T. ; Hasegawa, K. ; Saitou, A. ; Ishikawa, R. ; Honjou. Spatial modulation module consisting of a microstrip array antenna and dual scatterers for wireless power transmission. Antennas and Propagation (ISAP), 2012 International Symposium on

[15] Zhu Xi ; Zhang Xiaodong ; Wu Qingyu. Wireless Charging System Based on Switched Beam Smart Antenna Technique. Microwave, Antenna, Propagation and EMC Technologies for Wireless Communications, 2008 International Symposium on. 